\title{
INTERÉS GENERATIVO EN CUIDADORES DE PACIENTES CRÓNICOS, ADULTOS MAYORES Y JÓVENES UNIVERSITARIOS
}

\author{
Karina Reyes-Jarquín, Ana Luisa González-Celis Rangel, María Leticia Bautista-Díaz, Centli Guillén \\ Díaz Barriga, Ma. de Jesús Cruz Peralta, Violeta Alejandra Mendoza Madrigal, Laura Esmeralda \\ Raya Ayala y María Fernanda Casado Álvarez \\ Universidad Nacional Autónoma de México, Facultad de Estudios Superiores IztacalaMéxico
}

\begin{abstract}
RESUMEN
La generatividad es el interés del ser humano por guiar, compartir conocimientos, apoyar futuras generaciones y dejar un legado que le sobreviva. Desde la teoría de Erikson esta capacidad se presentaba en la madurez, siendo la paternidad la principal actividad generativa. No obstante, investigaciones posteriores indican que podría manifestarse también en la vejez, además de poner en duda si la actividad del cuidado indica alto interés generativo o presencia de estrés elevado, y el desconocimiento de si generaciones más jóvenes pueden desarrollar interés generativo. El objetivo de esta investigación es evaluar el interés generativo en cuidadores de pacientes crónicos (CPC), adultos mayores ( $\mathrm{AM}$ ) y jóvenes universitarios (JU), así como determinar las diferencias entre dichos grupos. Los resultados indican que los AM mostraron índices más altos en el puntaje global en comparación con los $\mathrm{JU}$ ( $\mathrm{z}=2.51, \mathrm{p}<0.05$ ) y con los $C P C(z=4.81, p<0.01)$. En el caso de los JU tuvieron altos niveles en dos dimensiones del interés generativo. En este sentido, aunque los AM y CPC son proveedores de cuidados, la generatividad parece desarrollarse de manera diferencial en ambos grupos debido a los motivos para desempeñar dicha función; aparentemente el interés generativo se puede desarrollar desde generaciones más jóvenes.
\end{abstract}

Palabras Clave:

generatividad, cuidadores, adultos mayores.

\section{GENERATIVE INTEREST IN CAREGIVERS OF CHRONIC PATIENTS, OLDER ADULTS AND YOUNG UNIVERSITY STUDENTS}

\begin{abstract}
Generativity is the interest of the human being to guide, share knowledge, support future generations and leave a legacy that will survive. From Erikson's theory, this capacity appeared in maturity, with fatherhood being the main generative activity. However, subsequent research indicated that it could also manifest itself in old age, in addition to questioning whether the care activity indicated high generative interest or the presence of high stress and, finally, the lack of knowledge if younger generations can develop generative interest. The objective of this research was to evaluate the generative interest in caregivers of chronic patients (CPC), older adults (AM) and young university students (JU), as well as to determine the differences between these groups. The results indicate that the AMs showed higher scores in the global score compared to the $J U(z=2.51, p<0.05)$ and with the CPC ( $z=4.81$, $p<0.01$ ). In the case of the JUs, they had high scores in two dimensions of generative interest. In this sense, although the AM and CPC are care providers, generativity seems to develop differently in both groups due to the reasons for performing this role; and apparently generative interest can develop from younger generations.
\end{abstract}

Keywords:

generativity, caregivers, elderly, university students.

Bitácora del Artículo:

| Recibido: 11 de Marzo de 2021 | Aceptado: 05 de mayo de 2021 | Publicado en línea: Enero - Junio de 2021 | 


\title{
INTERÉS GENERATIVO EN CUIDADORES DE PACIENTES CRÓNICOS, ADULTOS MAYORES Y JÓVENES UNIVERSITARIOS
}

\author{
Karina Reyes-Jarquín, Ana Luisa González-Celis Rangel, María Leticia Bautista-Díaz, Centli Guillén \\ Díaz Barriga, Ma. de Jesús Cruz Peralta, Violeta Alejandra Mendoza Madrigal, Laura Esmeralda \\ Raya Ayala y María Fernanda Casado Álvarez \\ Universidad Nacional Autónoma de México \\ México
}

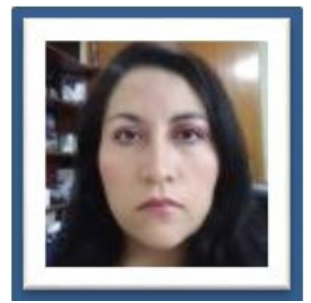

Karina Reyes-Jarquín.

FES- Iztacala, UNAM

Correo: krjarquin@gmail.com

Doctora en Psicología en UNAM-FESI. Es profesora de tiempo completo en la UNAM-FESI Pertenece al Sistema Nacional de Investigadores. Cuenta con dirección y co-dirección de tesis de licenciatura y maestría, publicaciones nacionales e internacionales, así como ponencias en eventos nacionales e internacionales.

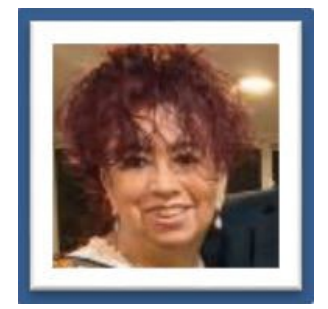

Ana Luisa González-Celis Rangel FES Iztacala, UNAM

Correo:algcr10@hotmail.com

Doctora en psicología por la Facultad de Psicología de la UNAM. Profesora de tiempo completo en la carrera de Psicología y Posgrado e Investigación de la UNAM-FESI, cuenta con el Nivel "D" en el PRIDE de la UNAM. Miembro del Sistema Nacional de Investigadores Nivel I. Ha sido reconocida con el Mérito Académico y distinguida con la Cátedra Alexander I. Oparin, en la FES Iztacala

\section{CONTRIBUCIÓN DE LOS AUTORES}

Karina Reyes-Jarquín estuvo a cargo de la concepción inicial del estudio, búsqueda de la literatura, trabajo de campo, análisis de datos, redacción de manuscrito. | Ana Luisa González-Celis Rangel participó en el análisis de datos, revisión del manuscrito, contribución intelectual.| María Leticia Bautista-Díaz contribuyó con la revisión de la literatura, revisión del manuscrito, contribución intelectual.| Centli Guillén Díaz Barriga participó en la revisión de la literatura, revisión del manuscrito, contribución intelectual.| María de Jesús Cruz Peralta apoyó en el trabajo de campo, revisión del manuscrito, contribución intelectual. | Violeta Alejandra Mendoza Madrigal contribuyó en el trabajo de campo, revisión del manuscrito, contribución intelectual. | Laura Esmeralda Raya Ayala revisó el manuscrito. | María Fernanda Casado Álvarez participó en el trabajo de campo y revisión del manuscrito

\section{AGRADECIMIENTOS}

La investigación forma parte del proyecto PAPIIT IN311019 de la DGAPA-UNAM, del cual se obtuvo financiamiento de manera parcial

\section{DAtos de Filiación de los Autores}

Facultad de Estudios Superiores Iztacala, UNAM

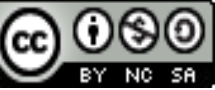

Copyright: (c) 2021 Reyes-Jarquín, K.; González-Celis Rangel, A. L.; Bautista-Díaz, M. L.; Díaz Barriga, C. G.; Cruz-Peralta, M. J.; Mendoza-Madrigal, V. A.; Raya-Ayala, L. E.; y Casado Álvarez, M. F.

Este es un artículo de acceso abierto distribuido bajo los términos de la licencia Creative Commons Reconocimiento-NoComercial 4.0 Internacional, por lo que su contenido gráfico y escrito se puede compartir, copiar y redistribuir total o parcialmente sin necesidad de permiso expreso de sus autoras con la única condición de que no se puede usar con fines directamente comerciales y los términos legales de cualquier trabajo derivado deben ser los mismos que se expresan en la presente declaración. La única condición es que se cite la fuente con referencia a la Revista Digital Internacional de Psicología y Ciencia Social y a sus autoras. 


\section{TABLA DE CONTENIDO}

\section{INTRODUCCIÓN}

MÉTODO

Participantes, 352

Diseño, 352

Procedimiento, 352

Mediciones, 352

Análisis estadístico, 353

RESULTADOS

Discusión

CONCLUSIONES 


\section{INTRODUCCIÓN}

D e acuerdo con la teoría de Erikson el ser humano pasa por ocho etapas críticas desde la infancia hasta la vejez que tienen la finalidad de lograr la identidad personal. La resolución positiva de cada etapa permite el acceso a las siguientes; no obstante, si la crisis no se resuelve de manera satisfactoria continúa demandando energía y causando dificultades (Villareal, Hernández, Leal, Medina y Vázquez, 2020). La séptima etapa cronológicamente se presenta en la mediana edad (entre los 30 y 50 años) y se identifica como Generatividad versus Estancamiento; en ésta la tarea más importante es la de efectuar actos de cuidado y guía de la siguiente generación. De acuerdo con Erikson, la acción más generativa es la paternidad (Cornaccione, et al., 2012; Mendoza-Nuñez, Vivaldo-Martínez y Martínez-Maldonado, 2018).

Así, Erikson (1982) define la generatividad como la capacidad del ser humano adulto que implica un interés por guiar, compartir conocimientos y apoyar a futuras generaciones, pero sobre todo el interés por dejar un legado que le sobreviva a su muerte. La generatividad se puede expresar en actividades como la crianza de los hijos, el cuidado a personas dependientes, la formación de los jóvenes, la producción de bienes y servicios, el compromiso social y la participación cívica y política (Villar, López y Celdrán, 2013).

McAdams y St. Aubin (1992) definieron la generatividad como un constructo multifacético con el objetivo primordial del cuidado y promoción de las próximas generaciones compuesto por siete características principales.

- Demanda cultural, la cual se desarrolla mediante las expectativas y las oportunidades sociales.

- Deseo interno, que surge a partir del requerimiento de ser necesitado o como símbolo de la inmortalidad.

- Preocupación generativa, que implica dejar un legado a las siguientes generaciones.

- Creencia en la especie, que significa tener esperanza en las futuras generaciones, en el avance y mejoramiento de la vida humana.

- Compromiso, por medio de la proposición de objetivos y decisiones.

- Acción generativa, mediante crear, mantener, preservar u ofrecer.
- Narración personal, la historia personal de vida será la que encaminará las acciones generativas.

Por otra parte, Kotre (1984) identificó algunos tipos de generatividad: 1) comunitaria, que implica nutrir y cuidar de otros estableciendo vínculos con otras personas; 2) agéntica, que está relacionada con la extensión y fortalecimiento de la persona mediante el liderazgo, la producción o la actividad creativa; y 3), cultural, que se refiere a la transmisión de instrumentos e ideas propias de una cultura a las siguientes generaciones.

Además es importante distinguir entre "interés" y "comportamiento generativo"; el primero se refiere a la existencia de la idea o pensamiento de hacer algo en beneficio de los demás, mientras que el segundo consiste en efectuar la acción en beneficio de los otros. El comportamiento generativo puede ser también una herramienta para buscar el beneficio de todos y la evolución sin la pérdida de costumbres, historia y valores (Benito-Ballesteros y De la Osa, 2020).

Por tanto, ser generativo implica contribuir al bienestar de los demás dejando una aportación voluntaria que va más allá de uno mismo y de recibir una gratificación inmediata; es decir, la recompensa será a largo plazo, intangible, placentera y satisfactoria. De este modo, ser generativo no sólo implica contribuir a una mejora, sino también que la acción hecha dará sentido y propósito a las personas, generando una recompensa personal (Villar et al., 2013; Triado-Tur, 2018).

Como se ha mencionado, Erikson introdujo el concepto de generatividad para referirse al reto que caracteriza la etapa de la madurez, por lo que el estudio empírico de este constructo se enfocó en un inicio en la población adulta. En el modelo de Erikson la superación con éxito de esta etapa aportaba determinada cualidad al yo, fortaleciéndolo y aumentando la probabilidad de abordar con éxito los retos de las etapas siguientes (Villar, 2012).

No obstante, en años recientes se ha encontrado que el perfil de la población adulta ha cambiado, marcando una tendencia hacia una mayor esperanza de vida, disponer de más tiempo libre después de la jubilación, y por tanto la posibilidad de una mayor implicación en actividades que antes no se podían hacer.

En consecuencia, la idea de la generatividad en adultos mayores es un marco prometedor para estudiar los aspectos positivos de la vejez en varios sentidos porque supone la integración de múltiples conceptos referidos al buen envejecer, da sentido al concepto del envejecimiento activo, además de que la generatividad puede reforzar el bienestar y funcionamiento de la vida cotidiana de las personas adultas mayores (Arias e Iglesias-Parro, 2015; González-Celis y Raya-Ayala, 2017; Triado-Tur, 2018). 
Villar (2012) identificó los tres principales ámbitos generativos para los adultos mayores: 1) el trabajo remunerado; 2) el voluntariado o participación cívica, y 3) el cuidado a otras personas. En cuanto al trabajo remunerado se le considera como una de las principales fuentes de estatus e identidad, pudiendo favorecer el mantenimiento de la salud mental y bienestar psicológico. Respecto al voluntariado, ha sido relacionado con la presencia de una mejor salud física, menores síntomas de depresión, y mayor bienestar y autoestima. Por último, el rubro del cuidado se ha enfocado al papel de los abuelos como proveedores de cuidados a los nietos, siendo una manera de sentirse útiles, una experiencia de enriquecimiento, mejora y descubrimiento de nuevas capacidades.

De acuerdo con Villar (2012), otros tipos de cuidado - como el que se ofrece a personas con dependencia o pacientes crónicos - han sido menos estudiados desde el punto de vista de la generatividad. En particular, en el caso de los pacientes con enfermedades crónicas es primordial la presencia de un cuidador, quien debe desplegar una atención muy exigente y demandante, desarrollando conocimientos, competencias y habilidades que le permitan proporcionar un adecuado cuidado al paciente (Martínez, 2020).

Se tiene evidencia empírica referente a que los cuidadores tienden a descuidar sus propias necesidades, ya sea por falta de tiempo o por su alto nivel de dedicación a la atención del paciente que cuidan (Arias-Rojas et al, 2014; López, 2016; Nava, 2012). De manera tradicional la tarea del cuidador primario en estos casos se ha considerado como una gran fuente de estrés y de consecuencias negativas para la salud y el bienestar, desarrollando incluso sobrecarga, depresión, ansiedad, fatiga, aislamiento social, baja calidad de vida y problemas financieros (Rodríguez-Lombana y Chaparro-Díaz, 2020).

Sin embargo, Peterson (2002) desarrolló un estudio con mujeres en la edad adulta; los resultados indican que quienes se dedicaban al cuidado de otros tenían más intereses generativos, se sentían menos sobrecargadas, tenían más conocimientos de los servicios disponibles en la comunidad, contaban con mayor número de personas dispuestas a ayudarles y estaban más dispuestas a admitir que necesitaban apoyo emocional.

En este sentido parece haber una discrepancia entre si el ser cuidador evidenciaría a una persona generativa o si es una actividad generadora de estrés y complicaciones psicoemocionales. Al respecto, autores como Martinson (2006) y Morrow-Howell (2007) proponen investigar si el hecho de ser cuidador podría ser una barrera para la participación en otras actividades generativas debido a la alta carga de tiempo y recursos que se dedican al cuidado.
Por otro lado, algo que Ilama la atención del estudio empírico de la generatividad es la gran variedad de ámbitos del desarrollo adulto en los que aparece como dimensión relevante, pues se han efectuado estudios con enfoque en el desarrollo personal, los roles familiares, o incluso en el análisis del crecimiento psicológico posterior a la enfermedad (Bellizzi, 2004; Peterson, 2002; Zacarés y Serra, 2011). No obstante, no hay evidencia empírica de estudios que incluyan población más joven, es decir, quienes aún cronológicamente no han transitado por la séptima etapa — generatividad versus estancamiento- de la que habla Erikson en su teoría. Al respecto, la única información disponible se refiere a que la preocupación, el compromiso y las conductas generativas son más elevadas en la mediana edad que en la adultez temprana (Zacarés y Serra, 2011).

Por tanto, es de interés teórico y empírico evaluar si la generatividad puede presentarse en el otro extremo del ciclo vital, es decir, las generaciones más jóvenes, así como se ha hecho en el caso de la población adulta mayor, lo cual supondría una contribución al bien común de los entornos en que se participa, un refuerzo y enriquecimiento de las instituciones sociales y un importante aporte para la continuidad generacional, al tiempo que representa una posibilidad para el desarrollo y crecimiento personal (Villar y Serrat, 2014).

De esta manera, el objetivo de esta investigación es evaluar el interés generativo en tres grupos poblacionales identificados como cuidadores de pacientes crónicos (CPC), adultos mayores (AM) y jóvenes universitarios (JU), así como determinar las diferencias en el interés generativo y sus dimensiones entre dichos grupos.

De acuerdo con la literatura revisada, las hipótesis planteadas son las siguientes.

- Los CPC presentarán un interés generativo más alto que los AM.

- Los AM presentarán un interés generativo más alto que los JU.

- Los CPC presentarán un interés generativo más alto que los JU.

- En comparación con los AM, los CPC reportarán niveles más elevados en todas las dimensiones que componen el interés generativo.

- En comparación con los JU, Los AM reportarán niveles más elevados en todas las dimensiones que componen el interés generativo.

- En comparación con los JU, los CPC reportarán niveles más elevados en todas las dimensiones que componen el interés generativo. 


\section{MÉTodo}

\section{Participantes}

Este estudio se desarrolló con una muestra no probabilística, intencional y por cuotas de 278 participantes, con la siguiente distribución: 78 CPC, 100 AM y 100 JU. Su participación fue voluntaria, consentida e informada.

Los criterios de inclusión para cada uno de los subgrupos fueron los siguientes.

- CPC: Ser mayores de edad, tener al menos un año fungiendo como cuidador de algún paciente diabético o renal, que recibiera atención médica en una clínica pública y haber firmado el consentimiento informado.

- AM: Ser mayores de 65 años, ser usuarios de la clínica pública y haber firmado el consentimiento informado.

- JU: Ser estudiante de nivel licenciatura en una universidad pública y haber firmado el consentimiento informado.

El criterio de exclusión para todos los subgrupos fue encontrarse entratamiento psiquiátrico; el criterio de eliminación consistió en contestar menos de $90 \%$ de los instrumentos utilizados.

El grupo de CPC estuvo conformado por $71.1 \%$ de mujeres y $28.9 \%$ de hombres, en su mayoría casados $(52.6 \%)$ y con una media de 47.09 años (DE $=16.74)$. El grupo de AM estuvo conformado por $65 \%$ de mujeres y $35 \%$ de hombres, en su mayoría casados $(60 \%)$ y con una media de 69.54 años (DE =6.48). El grupo de JU estuvo conformado por $65 \%$ de mujeres y $35 \%$ de hombres, en su mayoría solteros $(83 \%)$ y con una media de 19.75 años (DE $=1.50)$.

\section{Diseño}

Se utilizó un diseño no experimental, transeccional dado que se hizo la recolección de datos en un momento determinado para los tres subgrupos indicados (Kerlinger y Lee, 2002).

\section{Procedimiento}

La recolección de datos se ciñó a los señalamientos éticos para la investigación en psicología, normadas por el Código Ético del Psicólogo (Sociedad Mexicana de Psicología [SMP], 2010). Se solicitaron los permisos necesarios en cada una de las instituciones involucradas, las cuales consistieron en los siguientes aspectos.

- Una clínica de primer nivel de atención donde acudían a recibir atención médica pacientes dia- béticos y renales, lo que permitió la selección de sus cuidadores principales.

- Una clínica de primer nivel de atención donde acudían a recibir atención médica adultos mayores.

- Una universidad pública, lo que permitió la selección de jóvenes universitarios.

Con base en los criterios de selección, se eligió a los participantes de cada grupo y se les explicó el objetivo del estudio. En una única sesión firmaron el consentimiento informado que daba constancia de su participación de manera voluntaria, la confidencialidad y la libertad para abandonar el estudio cuando lo desearan, sin que esto repercutiera de ningún modo en la atención ofrecida por la institución. Al final contestaron los instrumentos indicados previamente.

\section{Mediciones}

Ficha de datos sociodemográficos, en la cual se incluían datos generales del participante.

Escala de Generatividad de Loyola (EGL), desarroIlada por McAdams y St. Aubin (1992). Mide el interés generativo en población adulta. Consta de 20 reactivos con opciones de respuesta tipo Likert de cuatro puntos (0 "nunca", 1 "ocasionalmente", 2 "muy a menudo" y 3 "casi siempre"); hay seis reactivos redactados en sentido negativo (reactivos 2, 5, 9, 13, 14 y 15) que deben recodificarse antes de obtener índices parciales y totales. El índice total puede tener un nivel de 0 a 60 puntos, donde un mayor nivel indica mayor interés generativo; además se calculan índices para cada una de sus cinco dimensiones.

- Transmitir conocimientos o habilidades (reactivos $1,3,12$ y 19).

- Hacer contribuciones significativas a la comunidad (reactivos 5, 15, 18 y 20).

- Hacer cosas que serán recordadas y dejar legado (reactivos 4, 6, 8, 10, 13 y 14).

- Ser creativo o productivo (reactivos 7 y 17).

- Tener responsabilidad hacia otras personas (reactivos 2, 9, 11 y 16 ).

Para una mejor comprensión de los resultados, en este estudio se transformaron los niveles de cada dimensión y el índice total a una escala del 0 a 100.

Según lo informado por McAdams y St. Aubin (1992), la EGL tiene una buena confiabilidad, indicada mediante un alfa de Cronbach de 0.83 que muestra de una alta consistencia interna, además de una fiabilidad test-retest con intervalo de tres semanas con una $r$ $=0.73$. En cuanto a la validez, se establecieron correlaciones positivas con medidas similares que evaluaban 
la misma variable, con lo cual se concluye que la EGL presenta una aceptable validez de constructo de tipo concurrente (McAdams, St. Aubin y Logan, 1993).

\section{Análisis estadístico}

Para efectuar el análisis estadístico se utilizó el paquete SPSS versión 22. Se hizo un análisis de varianza de Kruskal-Wallis para comparar los tres grupos; además, mediante la prueba $U$ de Mann-Whitney se hicieron comparaciones entre dos grupos independientes para probar en qué grupos había diferencias estadísticamente significativas.

\section{Resultados}

Para el total del interés generativo y sus dimensiones se obtuvieron las medianas como medida de tendencia central, tal como se muestra en la tabla 1.

En la tabla 2 se muestran los resultados obtenidos mediante el análisis Kruskal Wallis con el que se comparan los resultados de interés generativo entre los tres grupos poblacionales. Se observa que existieron diferencias estadísticamente significativas entre los tres grupos para el "Total del interés generativo" y todas las dimensiones que componen el instrumento.

Además se muestran los resultados de la prueba $U$ de Mann-Whitney con la que se hicieron las comparaciones entre AM-JU, CPC-JU y CPC-AM para cada dimensión y el "Total de interés generativo".

Se muestra que para el "Total del interés generativo" hubo diferencias estadísticamente significativas entre los CPC, AM y JU, indicando un interés más alto en los AM. Resultados similares se indican con las di- mensiones "Transmitir conocimientos o habilidades", "Hacer contribuciones significativas a la comunidad" y "Hacer cosas que serán recordadas y dejar legado"; en todas ellas hubo diferencias estadísticamente significativas, lo que demuestra un mayor nivel en los AM.

No obstante, en la dimensión "Tener responsabilidad hacia otras personas" hubo diferencias estadísticamente significativas en la comparación de los tres grupos, mostrando niveles más altos en los JU, mientras que en la dimensión "Ser creativo o productivo" se identificó que los JU tienen índices menores que los CPC y los AM.

\section{Discusión}

El objetivo de este estudio fue evaluar el interés generativo en tres grupos poblacionales identificados como cuidadores de pacientes crónicos (CPC), adultos mayores (AM) y jóvenes universitarios (JU), así como determinar las diferencias en el interés generativo y sus dimensiones entre dichos grupos.

En términos generales se encontraron diferencias estadísticamente significativas en el interés generativo entre los tres grupos poblacionales, mostrando beneficio para los AM participantes del estudio.

El grupo de AM reportó mayor interés generativo global, además de obtener los niveles más altos en dos de las cinco dimensiones del instrumento EGC. Las dimensiones indicadas fueron "Transmitir conocimiento o habilidades" y "Ser creativo o productivo". Dichos resultados son consistentes con lo informado por Villar et al., (2013), quienes afirman que el interés y las actividades generativas parecen seguir presentándose durante la

Tabla 1.

Medianas para el total del interés generativo y sus dimensiones.

\begin{tabular}{|c|c|c|c|c|c|c|}
\hline & \multicolumn{2}{|c|}{$\begin{array}{l}\text { CUIDADORES PACIENTES } \\
\text { CRÓNICOS (CPC) }\end{array}$} & \multicolumn{2}{|c|}{$\begin{array}{l}\text { AdULTOS MAYORES } \\
\text { (AM) }\end{array}$} & \multicolumn{2}{|c|}{$\begin{array}{l}\text { JóVENES UNIVERSITARIOS } \\
\text { (JU) }\end{array}$} \\
\hline & $\mathbf{N}$ & Mediana & $\mathbf{N}$ & Mediana & $\mathbf{N}$ & Mediana \\
\hline Total del interés generativo. & 77 & 55.00 & 88 & 68.33 & 95 & 65.00 \\
\hline $\begin{array}{l}\text { Transmitir conocimientos o } \\
\text { habilidades. }\end{array}$ & 78 & 66.66 & 98 & 75.00 & 98 & 66.66 \\
\hline $\begin{array}{l}\text { Hacer contribuciones significativas } \\
\text { a la comunidad. }\end{array}$ & 78 & 50.00 & 98 & 66.66 & 99 & 66.66 \\
\hline $\begin{array}{l}\text { Hacer cosas que serán recordadas y } \\
\text { dejar legado. }\end{array}$ & 77 & 55.55 & 95 & 66.66 & 100 & 61.11 \\
\hline Ser creativo o productivo. & 78 & 83.33 & 95 & 83.33 & 100 & 66.66 \\
\hline $\begin{array}{l}\text { Tener responsabilidad hacia otras } \\
\text { personas. }\end{array}$ & 78 & 50.00 & 97 & 66.66 & 99 & 66.66 \\
\hline
\end{tabular}


tercera edad, lo que pone en tela de juicio la asociación entre la generatividad y la mediana edad.

\section{Tabla 2}

Pruebas Kruskal Wallis y U de Mann-Whitney.

\begin{tabular}{|c|c|c|}
\hline VARIABLES & $\begin{array}{c}\text { KRUSKAL } \\
\text { WALLIS } \\
\text { JI CUADRADA }\end{array}$ & $\begin{array}{l}\text { U de ManN-Whitney } \\
\text { ComparaCiOnes } \\
\text { Significativas }\end{array}$ \\
\hline
\end{tabular}

\begin{tabular}{|c|c|c|}
\hline $\begin{array}{l}\text { Total del interés } \\
\text { generativo. }\end{array}$ & $24.44^{* *}$ & $\begin{array}{r}A M<J U\left(z=2.51^{*}\right) \\
C P C>J U\left(z=2.81^{* *}\right) \\
C P C>\operatorname{AM}\left(z=4.81^{* *}\right)\end{array}$ \\
\hline $\begin{array}{l}\text { Transmitir } \\
\text { conocimientos o } \\
\text { habilidades. }\end{array}$ & $15.32^{* *}$ & $\begin{array}{r}A M<J U\left(z=3.16^{* *}\right) \\
C P C>\operatorname{AM}\left(z=2.86^{* *}\right)\end{array}$ \\
\hline $\begin{array}{l}\text { Hacer } \\
\text { contribuciones } \\
\text { significativas a la } \\
\text { comunidad. }\end{array}$ & $15.95^{* *}$ & $\begin{array}{c}C P C>J U\left(z=3.07^{* *}\right) \\
C P C>\operatorname{AM}\left(z=3.74^{* *}\right)\end{array}$ \\
\hline $\begin{array}{l}\text { Hacer cosas que } \\
\text { serán recordadas } \\
\text { y dejar legado. }\end{array}$ & $23.53^{* *}$ & $\begin{array}{r}A M<J U\left(z=3.55^{* *}\right) \\
C P C>A M\left(z=4.56^{* *}\right)\end{array}$ \\
\hline $\begin{array}{l}\text { Ser creativo o } \\
\text { productivo. }\end{array}$ & $19.15^{* *}$ & $\begin{aligned} A M & <J U\left(z=4.21^{* *}\right) \\
C P C & <J U\left(z=3.06^{* *}\right)\end{aligned}$ \\
\hline $\begin{array}{l}\text { Tener } \\
\text { ressponsabilidad } \\
\text { hacia otras } \\
\text { personas. }\end{array}$ & $30.47^{* *}$ & $\begin{array}{r}A M>J U\left(z=2.62^{* *}\right) \\
C P C>J U\left(z=5.32^{* *}\right) \\
C P C>\operatorname{AM}\left(z=3.36^{* *}\right)\end{array}$ \\
\hline
\end{tabular}

AM (adultos mayores), JU (jóvenes universitarios), CPC (cuidadores de pacientes crónicos).

${ }^{*} \mathrm{p}<0.05 .{ }^{* *} \mathrm{p}<0.01$.

Incluso el mismo Erikson acuñó el término "grand generativity" para reconocer que los adultos mayores también se ven inmersos en actividades generativas ayudando a otros efectuando funciones de paternidad, mentoría o amistad, además de expresar su interés por compartir conocimientos y valores a futuras generaciones (Erikson et al.,1986).

Por otra parte, se quería conocer si generaciones más jóvenes podían desarrollar interés generativo, encontrando que el grupo de JU reportó niveles altos (similares a los de los AM) en dimensiones como "Hacer contribuciones significativas a la comunidad" y "Tener responsabilidad hacia otras personas"; además, es necesario indicar que en el interés generativo global este grupo obtuvo niveles más altos que los CPC. Estos resultados son reveladores, dado que podrían indicar que la generatividad se presenta incluso en edades más tempranas, lo cual es prometedor para el mejoramiento social por medio de las acciones que los jóvenes y adultos jóvenes puedan efectuar.
En este sentido, es necesario recordar que las personas generativas se caracterizan por mostrar genuino interés en el desarrollo de generaciones jóvenes, en el campo laboral seleccionado y en la sociedad que heredarán; por lo general fungen como guías y expresan la necesidad de compartir aprendizajes y experiencias (Cornaccione et al., 2012). En el caso de la muestra formada por jovenes universitarios, los resultados sugieren que pudieran estar interesados en el crecimiento de sus propios pares, en el área laboral en que incursionarán después de sus estudios universitarios y en el futuro de la sociedad en que se desarrollan.

Existe una discrepancia en la función de los cuidadores frente a la generatividad, dado que hay evidencia teórica y empírica previa que indica un alto interés generativo, pero también hay estudios que indican la presencia de estrés y sobrecarga. En este sentido, en este estudio el grupo de CPC obtuvo los niveles más bajos en la mayoría de las dimensiones del instrumento EGC (excepto "Ser creativo o productivo"), así como en el interés generativo global. Estos resultados son consistentes con lo indicado por Villar (2012), que refiere que la tarea de los cuidadores primarios es una fuente de estrés y de consecuencias negativas para la salud y el bienestar.

Asimismo, es necesario indicar que las actividades generativas, además de contribuir al bien común, deben incrementar la satisfacción de las personas que las efectúan; no obstante, de acuerdo con Villar, Celdrán, Fabá y Serrat (2013), las actividades de cuidado parecen no cumplir con estos requisitos porque quien las desarrolla no presenta mayores niveles de bienestar que quienes no lo hacen. Entonces, la ausencia de beneficios personales podría evidenciar que la motivación para efectuarlas no es un interés generativo genuino, sino un contexto de obligación o falta de otras opciones.

Así, parece que aunque los AM y los CPC desarroIlan actividades de cuidado, la generatividad se efectúa de manera diferencial en ambos grupos, lo cual se explicaría por los factores que los motivan a desempeñar dicha función. En el caso de los CPC parece una cuestión de obligación, mientras que los AM parecen tener mayor libertad al respecto, además de que existe evidencia de estar relacionado con una mejor calidad de vida sobre todo en autonomía, participación social e intimidad (González-Celis y Mendoza, 2016), lo cual es consistente con lo indicado por Martinson (2006) y Morrow-Howell (2007), quienes consideran que ser cuidador podría interferir con el desarrollo de otras actividades generativas porque un cuidador utiliza gran parte de su tiempo y recursos en ejercer su labor. 
Además podría influir el nivel de tiempo y exigencia necesarios para cada cuidado; en el caso de los CPC tienen que enfrentarse al cuidado de una persona dependiente, con un funcionamiento físico y cognitivo deteriorado y que requiere un seguimiento médico especial, tal como lo indica Martínez (2020), mientras que los AM desarrollan funciones de cuidado a los nietos que por lo general no tienen ningún requerimiento especial como los pacientes crónicos.

En este sentido, Villar (2012) indica que ser abuelos y estar al tanto del cuidado de los nietos representa una excelente manera de ser generativo para los adultos mayores; lo anterior porque un abuelo puede proporcionar atención y cuidado cuando por alguna circunstancia los padres no pueden hacerlo de manera completa.

\section{Conclusiones}

Este estudio representa un acercamiento al caso particular de la sociedad mexicana, e incluso latinoamericana, donde los CPC que se enfrentan al cuidado de sus familiares lo hacen sin tener la formación necesaria para hacerlo, lo cual puede derivar en el bajo interés por hacerlo; en el caso de los AM es necesario investigar cuáles son los comportamientos generativos que propicien que contemplen una recompensa intangible, placentera y satisfactoria. Respecto a los JU sería muy recomendable fomentar el cuidado de otras generaciones una vez que se sabe que el interés generativo está presente en este grupo.

Los hallazgos de esta investigación aportan evidencia empírica al campo de la generatividad, aunque hay limitaciones que es necesario enunciar y que futuras investigaciones retomen. En primer lugar habría que diversificar la muestra para incluir distintos grupos etarios e incluso cuidadores de personas con diversos padecimientos, como dependencia física, deterioro cognitivo y enfermedades crónicas, entre otros. En segundo lugar sería recomendable la evaluación tanto del interés generativo como de las acciones generativas que se hacen con el objetivo de comprobar si los AM son generativos mediante el cuidado de nietos o si hay algún otro comportamiento generativo con mayor presencia e impacto. Por último, sería deseable evaluar la función de la generatividad como factor protector para la ausencia de la sobrecarga de los CPC, e incluso desarrollar intervenciones al respecto.

\section{Referencias}

Arias-Rojas, M., Barrera-Ortiz, L., Mabel-Carrillo, G., Chaparro-Díaz, L., Sánchez-Herrera, B., \& Vargas-Rosero, E. (2014). Cuidadores familiares de personas con enfermedad crónica en las regiones de frontera colombiana: Perfil y carga percibida de cuidado. Revista Facultad de Medicina, 62(3), 387-397. http:// dx.doi.org/10.15446/revfacmed.v62n3.39091

Arias, A., \& Iglesias-Parro, S. (2015). La generatividad como una forma de envejecimiento exitoso. Estudios del efecto mediacional de los vínculos sociales. European Journal of Investigation in Health. 5(1), 109-120. https://doi. org/10.30552/ejihpe.v5i1.94

Bellizzi, K. M. (2004). Expressions of generativity and posttraumatic growth in adult cancer survivors. The International Journal of Aging and Human Development, 58, 267-287. https://doi.org/10.2190/DC07-CPVW4UVE-5GK0

Benito-Ballesteros, Á., \& De la Osa, I. (2020). La generatividad a través de la cultura. Una revisión sistemática. Tendencias Sociales Revista de Sociología, 5, 63-79. https://doi. org/10.5944/ts.5.2020.27748

Cornaccione, M., Urrutia, A., Ferragut, L., Moiseet, G., Guzmán, E., \& Sesma, S. (2012). Validez y estabilidad de la Escala Multidimensional de Generatividad (EMG). Anuario de Investigaciones de la Facultad de Psicología, 1(1), 218-228.

Erikson, E. (1982). The life cycle completed. Nueva York: Norton. Erikson, E., Erikson, J., \& Kivnick, H. (1986). Vital involvement in old age. Nueva York: Norton. https://doi. org/10.1177/074171368904000107

González-Celis, A., \& Mendoza, V. (2016). Comportamiento generativo y su efecto en la calidad de vida en adultos mayores. Revista Electrónica de Psicología Iztacala, 19(4), 1333-1354

González-Celis, A., \& Raya-Ayala, L. (2017). Generatividad en adultos mayores mexicanos: Asociación entre interés y comportamientos generativos. Psicología y Salud, 27(1), 79-88.

Kerlinger, F., \& Lee, H. (2002). Investigación del comportamiento. Métodos de investigación en ciencias sociales. México: McGraw-Hill.

Kotre, J. (1984). Outliving the self: Generativity and the interpretation of lives. Baltimore: John Hopkins University Press.

López, E. (2016). Puesta al día: Cuidador informal. Revista de Enfermería Castilla y León, 8(1), 71-77.

Martínez, L. (2020). El cuidador de pacientes con enfermedad crónica discapacitante: Descripción, análisis e implicaciones para la gestión (tesis doctoral). Universidad del Rosario.

Martinson, M. (2006). Opportunities or obligations? Civic engagement and older adults. Generations, 30(4), 59-65. https://doi.org/10.1093/geront/46.3.318

McAdams, D., \& St. Aubin, E. (1992). A theory of generativity and its assessment through self-report, behavioral acts, and narrative themes in autobiography. Journal of Personality and Social Psychology, 62, 1003-1015. https://doi. org/10.1037/0022-3514.62.6.1003 
McAdams, D., St. Aubin, E., \& Logan, R. (1993). Generativity among young, midlife, and older adults. Pyschology and Aging, 8, 221-230. https://doi.org/10.1037/08827974.8.2.221

Mendoza-Nuñez, V., Vivaldo-Martínez, M., \& MartínezMaldonado, M. (2018). Modelo comunitario de envejecimiento saludable enmarcado en la resiliencia y la generatividad. Revista Médica del Instituto Mexicano del Seguro Social, 56(1), 1-13.

Morrow-Howell, N. (2007). A longer worklife: The new road to volunteering. Generations: Journal of the American Society on Aging, 31(1), 63-67. https://doi. org/10.1177/0899764015602128

Nava, G. M. (2012). La calidad de vida: análisis multidimensional. Revista Enfermería Neurológica, 11(3), 129-137.

Peterson, B. (2002). Longitudinal analysis of midlife generativity, intergenerational roles, and caregiving. Psychology and Aging, 17(1), 161-168. https://doi.org/10.1037/08827974.17.1.161

Rodríguez-Lombana, L., \& Chaparro-Díaz, L. (2020). Soporte social y sobrecarga en cuidadores: Revisión integrativa. Revista Cuidarte, 11(1), 1-13, http://dx.doi. org/10.15649/cuidarte.885

Sociedad Mexicana de Psicología (SMP) (2010). Código ético del psicólogo. México: Trillas.

Triado- Tur, C. (2018). Envejecimiento activo, generatividad y aprendizaje. Aula Abierta, 47(1), 63-66.

Villar, F. (2012). Hacerse bien haciendo el bien: La contribución de la generatividad al estudio del buen envejecer. Información psicológica, 104, 39-56.

Villar, F., Celdrán, M., Fabá J., \& Serrat, R. (2013). La generatividad en la vejez: Extensión y perfil de las actividades generativas en una muestra representativa de personas mayores españolas. Revista Ibero-Americana de Gerontología, 21, 61-79.

Villar, F., López, O., \& Celdrán M. (2013). La generatividad en la vejez y su relación con el bienestar: ¿Quien más contribuye es quien más se beneficia? Anales de Psicología, 29(3), 897906. http://dx.doi.org/10.6018/analesps.29.3.145171

Villar, F., \& Serrat, R. (2014). La participación cívica de las personas mayores como expresión de la generatividad en la vejez. Estudios Interdisciplinarios, Porto Alegre, 19(2), 345-358. https://doi.org/10.22456/2316-2171.44952

Villareal, M., Hernandiz, J., Leal, C., Medina, S., \& Vázquez, C. (2020). Bienestar psicológico y Generatividad en adultos mayores que practican el voluntariado. Neurama Revista Electrónica de Psicogerontología, 7(1), 46-57.

Zacarés, J. J., \& Serra, E. (2011). Explorando el territorio del desarrollo adulto: La clave de la generatividad, Cultura y Educación 23(1), 75-88. https://doi. org/10.1174/113564011794728533 


\section{Meta-Análisis del Artículo}




\section{Dimensión Cuantitativa}

\section{Perfil de Evaluación entre pares}
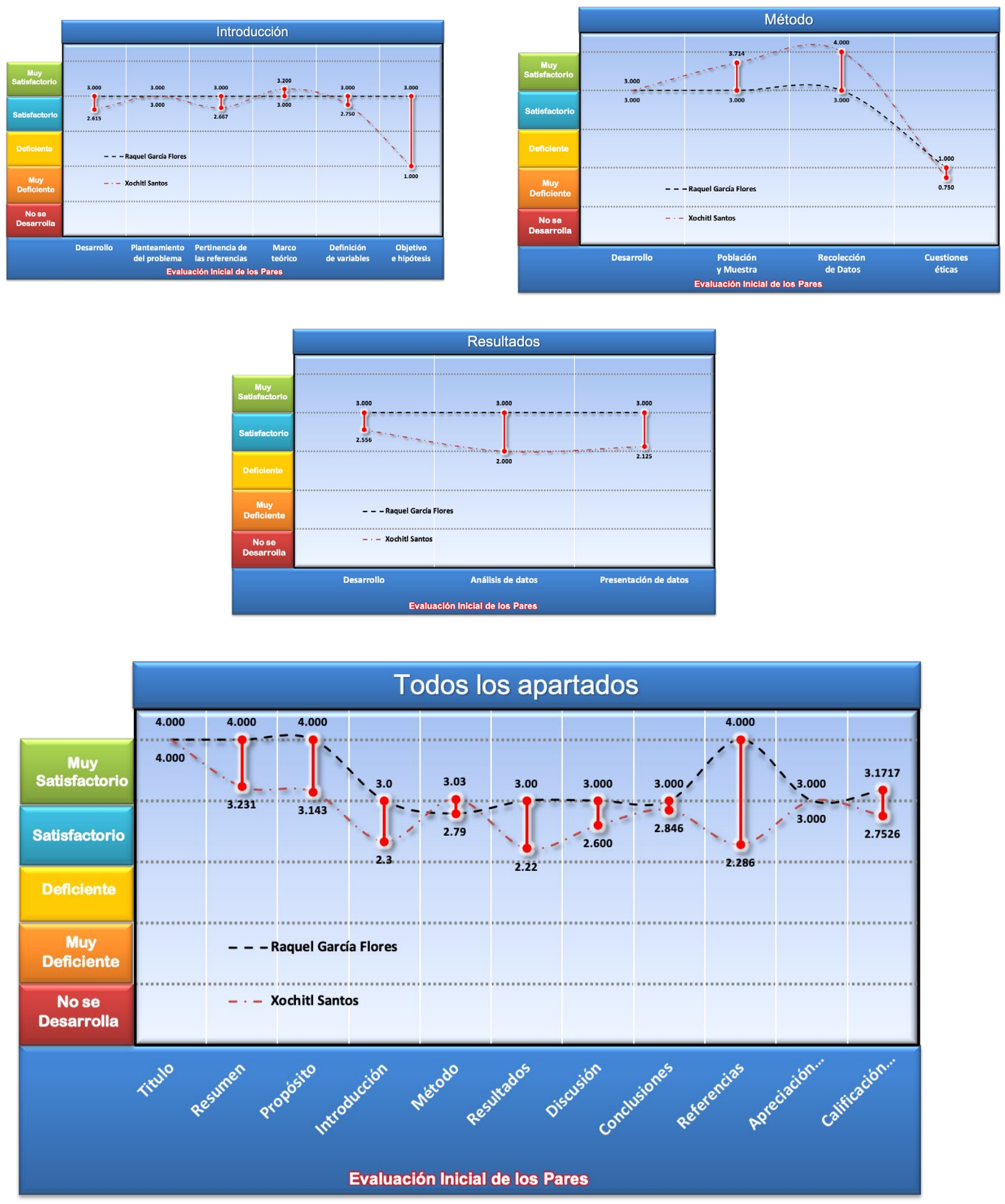


\section{Índice de Concordancia}

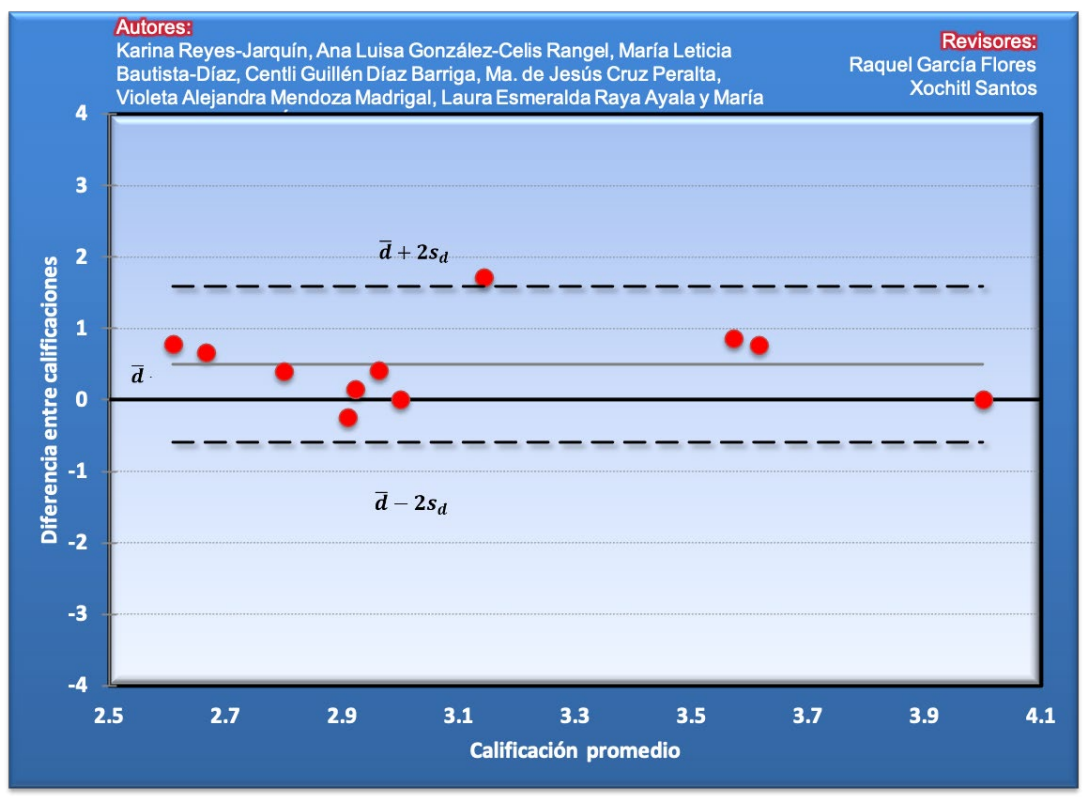

\section{Índice de Acuerdo}

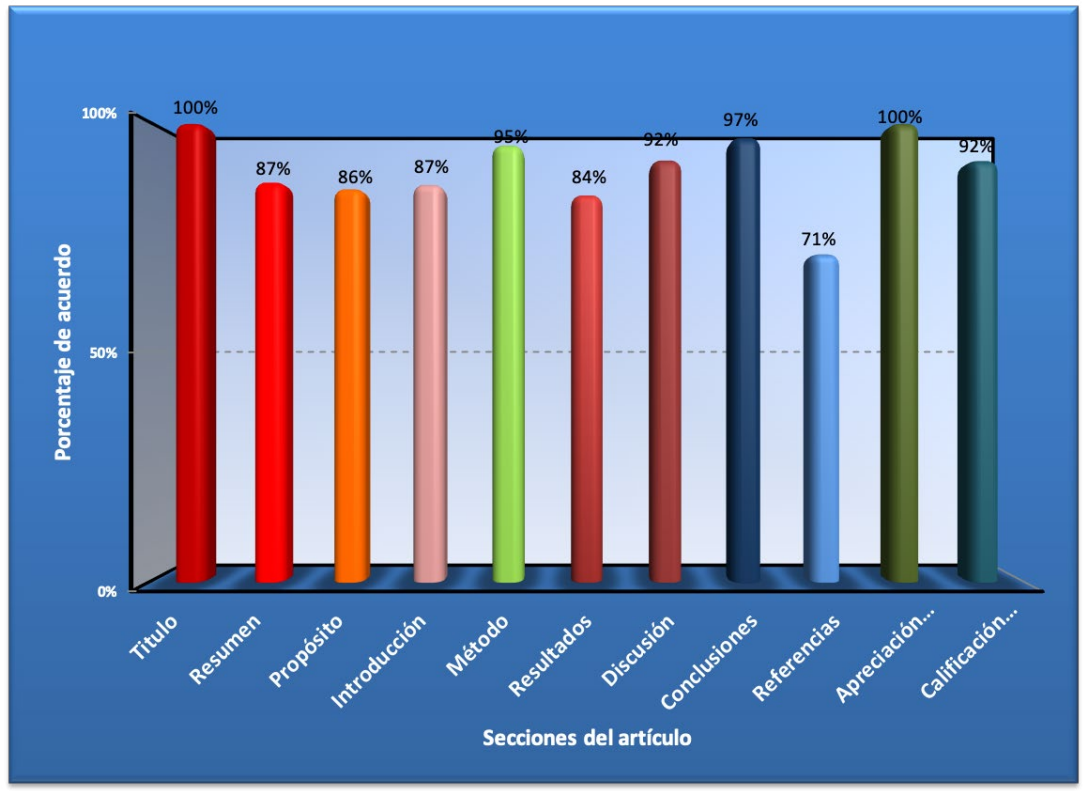




\begin{tabular}{|c|c|}
\hline Revisor 1 & Revisor 2 \\
\hline Xochitl Santos & Raquel García Flores \\
\hline \multicolumn{2}{|c|}{ Título/Autoría } \\
\hline $\begin{array}{l}\text { El título es adecuado y refleja el objetivo del estudio, es } \\
\text { breve y conciso; se ajusta a los requerimientos de la revista. }\end{array}$ & \\
\hline \multicolumn{2}{|c|}{ Resumen } \\
\hline $\begin{array}{l}\text { El resumen cumple con el criterio de número de pala- } \\
\text { bras, sin embargo, se encontraron algunos detalles que } \\
\text { podrían mejorar la calidad del mismo: En el primero } \\
\text { párrafo se hace hincapié en la Teoría que fundamenta } \\
\text { el constructo, sin embargo, se deja de fuera por qué es } \\
\text { importante evaluar el interés en el grupo de universi- } \\
\text { tarios (es parte fundamental del trabajo y del plantea- } \\
\text { miento del problema). Y en el último párrafo también } \\
\text { se excluyen los resultados en el grupo de jóvenes. Sería } \\
\text { conveniente que se incluyeran las implicaciones de los } \\
\text { hallazgos de este estudio, de manera breve y concisa. }\end{array}$ & \\
\hline \multicolumn{2}{|c|}{ Próposito del Estudio } \\
\hline $\begin{array}{l}\text { Sería importante ampliar el planteamiento del proble- } \\
\text { ma, justificar teórica y empíricamente por qué utilizar } \\
\text { estos tres grupos. Dado el alcance del estudio, podrían } \\
\text { agregar hipótesis de investigación. Incluso implicacio- } \\
\text { nes } C \text { implicaciones teóricas y prácticas del estudio. }\end{array}$ & \\
\hline \multicolumn{2}{|c|}{ Introducción } \\
\hline $\begin{array}{l}\text { Es importante que se agreguen las hipótesis de investiga- } \\
\text { ción. El tipo de estudio y alcance lo requiere. Sería impor- } \\
\text { tante que se profundice en por qué es importante inves- } \\
\text { tigar este tema en los tres grupos evaluados, aportar más } \\
\text { evidencia teórica y empírica del estudio de esta variable } \\
\text { en los tres grupos o bien explicar si no existe, la relevancia } \\
\text { de los hallazgos o implicaciones teóricas del este estudio. }\end{array}$ & en general la introducción me parece adecuada \\
\hline
\end{tabular}




\begin{tabular}{|l|ll}
\hline Revisor 1 & Revisor 2 \\
\hline & Método \\
\hline
\end{tabular}

Falta mencionar los criterios de exclusión y eliminación para el estudio. Podrían incluir si el protocolo fue aprobado por algún Comité de Ética. En el manuscrito se menciona que se obtuvieron los permisos de las instituciones el método se encuentra redactado con claridad implicadas, sin embargo, no queda claro de qué tipo de institución se esta hablado, ya que la muestra es diversa y se conforma por tres grupos poblacionales diferentes.

\section{Resultados}

Se sugiere a los autores plantear al menos una hipótesis de investigación dado el alcance del estudio. Los datos se analizaron con estadística paramétrica (ANOVA de un factor), sin embargo, no se cumplen los criterios para el uso de dicha prueba. Se recomienda utilizar otro estadístico para distribución no normal. Revisar el manual APA para formato de número de tablas (negristas). Incluir una nota en la que describan las abreviaturas (grupos a los que corresponden).

\section{Discusión}

Algunos párrafos están deben integrarse en el anterior para dar continuidad a la idea, por ejemplo: el tres y cuatro, seis y siete. Revisar la redacción del párrafo ocho. En el texto se debe revisar el uso de et al., (Manual APA). En la discusión los autores asumen hallazgos que no evaluaron como "que la tarea de los cuidadores primarios es una fuente de estrés y de consecuencias negativas para la salud y el bienestar". En general, se sugiere discutir con base en la literatura que, en este la proporción de mujeres que participaron en el estudio fue mucho mayor a la de los hombres, se sugiere incluir en la discusión este tema. Quizás los resultados de este estudio sean atribuibles a las mujeres, por lo que en un futuro se sugeriría llevar a cabo estudios para identificar diferencias entre hombres y mujeres. caso requiere mayor énfasis en los estudios previos de la variable de interés y en la unidad de análisis.

\section{Conclusiones}

Valdría la pena ahondar más desde la introducción a qué se refieren con cuidador, su importancia y los efectos del cuidado en contextos de enfermedad crónica. Hay estudios en los que se observa que los jóvenes también llegan a asumir el rol de cuidador en contexto de enfermedad crónica. En el párrafo de las limitaciones se menciona incluir otro tipo de cuidadores, ¿a cuáles se hace referencia?

se sugiere incluir como futuras líneas los estudios de género en relación al interés generativo. 
Artículo Editorial | Interés generativo en cuidadores de pacientes crónicos...| Reyes-Jarquín, et. al.

\begin{tabular}{|l|l|}
\hline \multicolumn{1}{|c|}{ Revisor 1 } & \multicolumn{1}{|c|}{ Revisor 2 } \\
\hline \multicolumn{2}{|c|}{ Referencias } \\
\hline $\begin{array}{l}\text { Revisar Manual APA para el uso de \& (dos autores). Re- } \\
\text { visar Manual APA uso de y o \& en textos en inglés. En } \\
\text { términos generales se sugiere a los autores actualizar sus } \\
\text { referencias y ampliar su marco teórico, ya que eso limita } \\
\text { su discusión y conclusiones. }\end{array}$ & las referencias están redactadas correctamente. \\
\hline
\end{tabular}

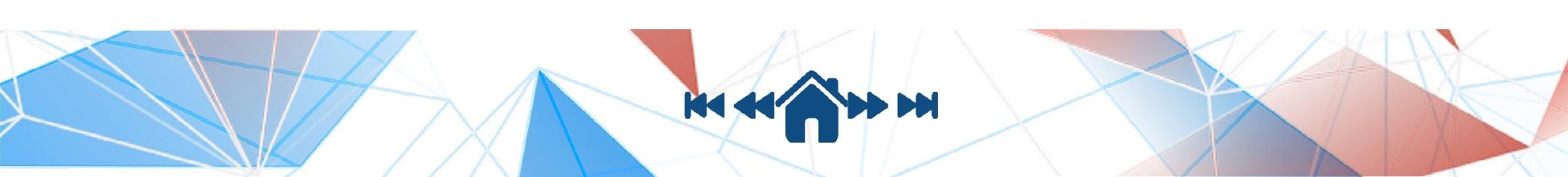


